\title{
EVALUATION OF BOVINE PARTURIENT PARESIS IN 64 COWS WITH RESPECT TO PREVENTIVE METHODS
}

\author{
Mehdi Sakha, Majid Jamshidian \\ Dept. Large Animal Internal Medicine, School of Veterinary Medicine, Shahid Bahonar University of \\ Kerman, Kerman, Iran. P.O BOX 76169-133,msakha@yahoo.com,msakha@arg3.ac.uk.ir
}

\section{Introduction}

Parturient paresis is an important metabolic disease occurring most commonly in high producing cows due to calcium depletion of cholestrum. Three stages have been described for the disease. The mark signs are general muscular weakness, circulatory collapse and depression.

\begin{abstract}
AIM
Milk fever is well known disease in metabolic diseases and almost there are no clinical signs that are obvious for veterinarians but the complete ethiology and the best way of prevention is controversial. The most important objective of this study is following these factors. Due to formal data the incidence of BPP in this area was high and it was one of the important compliant of owners, so we decided that follow the ethiologies and recommend the possible preventive methods.
\end{abstract}

\section{Method}

In this study 64 crossbred Holstein cows aged between 4-10 years were visited in either military veterinary clinic or private husbandry in city of Torbate-Heidarrieh in north -east of IRAN in nine months fromOCT.1999 to JUN 2000(time of the most parturition in the city). Physical exam were carried out carefully for each cases and a serum sample was sent to the laboratory for calcium level. Calcium was measured by commercial kit. Further it a full information of diet formula and dietary management was considered. Although it was not very important for us but we consider clinical signs and mode of treatment carefully, so we have now another confirmation of already register signs.

\section{Results and discussion}

From the 356 parturitions there was 64 case of BPP that is almost 18 percent incidence.

The total serum calcium were $2-4.5 \mathrm{mg} / \mathrm{dl} .29$ cases had $2-3.5 \mathrm{mg} / \mathrm{dl}$ and 35 cases had $3.5-4.5 \mathrm{mg} / \mathrm{dl}$ calcium level, with the increasing of age the serum calcium levels show decreasing rate. Decreasing of calcium level almost had direct correlation with the severity of signs.

The most clinical signs that cows showed were low temperature $36-38 \mathrm{c}(62$ cases $)$, increasing heart rate up to $80 \mathrm{bpm}$ (56 cases), mydriasis and poor reflex to PLR (62 cases), relaxation of anus (63 cases), sternal recumbency with s-shape neck (62 cases). Most cases were at second stage of disease (62 cases).

Most cases were between 6-9 years old and 4-7 parturition $(\mathrm{P}<0.05) .41$ cases of this study showed the disease at 48 hours after parturition $(\mathrm{P}<0.05)$.

Feeding ingredients include alfalfa hay, concentrate and wheat straw. 31 cases had only alfalfa in their feeding that is probably one of the factors responsible for the disease ( $>150 \mathrm{gr}$ calcium per day).

It seems that among several factors affect on milk fever in this city, type of feeding is more important and for prevention of disease, correction of diet and probably addition of anion salts is necessary. 